\title{
Una mezcla láctea fortificada con hierro y zinc mejora los niveles de hemoglobina en niños de 2 a 10 años en una comunidad andina en Perú: Ensayo controlado aleatorizado, doble ciego
}

\section{A dairy mixture fortified with iron and zinc improves hemoglobin levels in children aged 2 to 10 years in an andean community in Peru: Randomized, double-blind controlled trial}

\section{$\mathrm{DOI}$}

https://doi.org/10.35434/rcmhnaaa.2021.143.1250

\section{RESUMEN}

Introducción: Investigaciones anteriores coinciden en que el enriquecimiento de alimentos es la mejor estrategia a largo plazo en la prevención de la deficiencia de hierro. Nuestro objetivo es evaluar el efecto de una mezcla láctea fortificada con hierro y zinc sobre los niveles de hemoglobina en niños de 2 a 10 años. Material y métodos: Se realizó un ensayo clínico aleatorizado, doble ciego, controlado en una comunidad ubicada a 2,590 metros sobre el nivel del mar. Se incluyeron niños con niveles en el límite inferior de los rangos de normalidad o anemia leve. Asignamos a los participantes de manera aleatoria que recibieran la mezcla láctea fortificada con hierro y zinc o una mezcla láctea no fortificada. La mezcla láctea se administró cinco días a la semana durante 24 semanas. Nuestro resultado principal fue el nivel de hemoglobina corregida tomando en cuenta la altura sobre el nivel del mar y los índices antropométricos se evaluaron como resultado secundario. La magnitud de la diferencia observada entre los grupos se estimó mediante la prueba t de Student. Todos los análisis se realizaron por intención de tratar. Resultados: Se asignaron al azar 67 niños, intervención: $n=32$ y control: $n=35$. La edad media fue de 5,8 años; 30 eran mujeres. La hemoglobina al final del seguimiento fue $13,1 \mathrm{gr} / \mathrm{dL}(0,9)$ en el grupo experimental mientras que, en el grupo control, fue de $12,1 \mathrm{gr} / \mathrm{dL}(0,8) ; \mathrm{p}<0,001$. Asimismo, se evidenció una diferencia positiva en los indicadores antropométricos en el grupo experimental. Conclusión: La mezcla láctea fortificada con hierro y zinc aumenta los niveles de hemoglobina y los índices antropométricos en niños de 2 a 10 años.

Palabras Clave: Niño, Mezcla Láctea, Hierro, Zinc, Hemoglobina, Perú (Fuente: DeCS-BIREME).

\section{ABSTRACT}

Objetive: Previous research agrees that food fortification is the best long-term strategy for preventing iron deficiency. Our goal is to evaluate the effect of a dairy mixture fortified with iron and zinc on hemoglobin levels in children aged 2 to 10 years. Material and methods: A

Youmi Paz-Olivas ${ }^{1,2, a}$, Felipe L.Ignacio-Cconchoy ${ }^{3,4,5, b}$, Paola C.Meza-Flores ${ }^{1, c}$, Alexandra Acosta-Claros de Postigo ${ }^{1, c}$, Vicente A. Benites-Zapata ${ }^{4,5, d}$

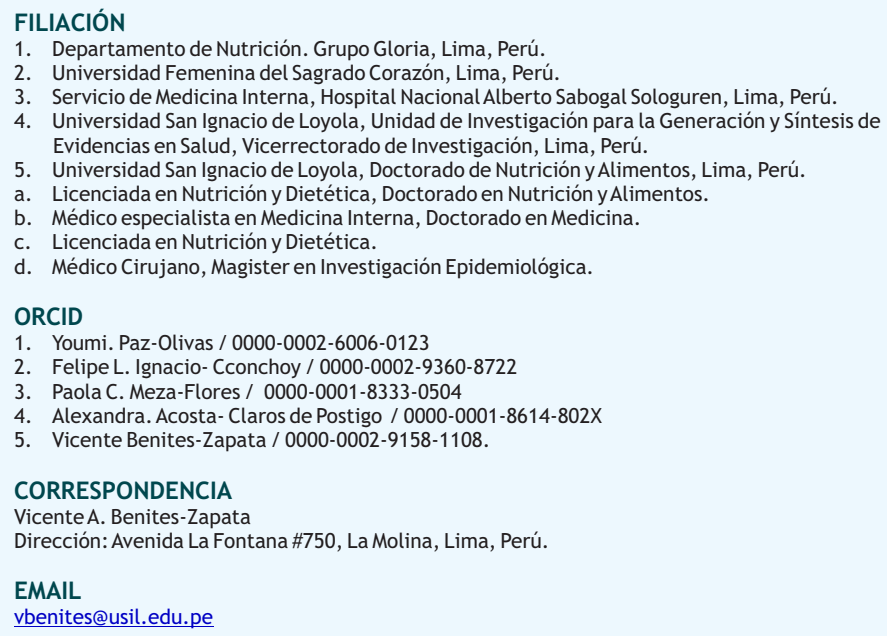
diseño del artículo, recolección de resultados, obtención de financiamiento, supervisión y administración del proyecto, redacción, revisión crítica del artículo y aprobación de la versión final. Vicente A. Benites Zapata y Felipe L. Ignacio-Cconchoy: Diseño del artículo, análisis estadístico e interpretación de los datos, redacción del primer borrador, revisión crítica del artículo y aprobación de la versión final

\section{REVISIÓN DE PARES}

Recibido: $17 / 06 / 202$

Aceptado: 20/09/2021

\section{COMO CITAR}

Paz-Olivas, Y., Ignacio-Cconchoy, F., Meza-Flores, P., Acosta-Claros de Postigo, A., \& BenitesZapata, $V$. Una mezcla láctea fortificada con hierro y zinc mejora los niveles de hemoglobina en niños de 2 a 10 años en una comunidad andina en Perú: Ensayo controlado aleatorizado, doble ciego. Revista Del Cuerpo Médico Hospital Nacional Almanzor Aguinaga Asenjo, 2021, 14(3), 304 -310. https://doi.org/10.35434/rcmhnaaa.2021.143.1250

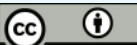

Esta obra está bajo una Licencia Creative Commons Atribución 4.0 Internacional. Versión Impresa: ISSN: 2225-5109

Versión Electrónica: ISSN: 2227-4731

Cross Ref. DOI: $10.35434 /$ rcmhnaaa

OJS: https://cmhnaaa.org.pe/ojs 
randomized, double-blind, controlled clinical trial was conducted in a community located 2,590 meters above sea level. Children with normal levels of hemoglobin or mild anemia were included. We assign participants to receive the iron-zinc-fortified dairy mixture or an unfortified dairy mixture. The supplement was administered five days a week for 24 weeks. Our main result was the corrected hemoglobin level taking into account the height above sea level and anthropometric indices were evaluated as a secondary result. The magnitude of the difference observed between the groups was estimated by the Student t-test. All analyzes were done with the intention to treat. Results: 67 children were randomly assigned, intervention: $n=32$ and control: $n=35$. The mean age was 5.8 years; 30 were women. Hemoglobin at the end of the follow-up was $13.1 \mathrm{~g} / \mathrm{dL}(0.9)$ in the experimental group, while, in the control group, it was $12.1 \mathrm{~g} / \mathrm{dL}(0.8) ; \mathrm{p}$ $<0.001$. Likewise, a positive difference was evidenced in the anthropometric indicators in the experimental group. Conclusion: Dairy mixture fortified with iron and zinc increases hemoglobin levels and anthropometric indices in children from 2 to 10 years old.

Keywords: Child, Dairy mixture, Iron, Zinc, Hemoglobins, Peru (Source: MeSH/NLM) .

\section{INTRODUCCIÓN}

La anemia es un problema de salud pública en todo el mundo y sigue siendo un problema grave en el Perú( ${ }^{(1)}$. La anemia está presente en un tercio de la población mundial y está liderada por la deficiencia de hierro ${ }^{(2)}$. En Perú, la anemia infantil afecta al 43,6\% de los niños y niñas de 6 a 36 meses, siendo más prevalente en los niños de 6 a 18 meses, en este grupo seis de cada diez niños presentan anemia ${ }^{(5)}$.

En Perú, el patrón de alimentación es el consumo de hierro de origen vegetal, se observa una ingesta diaria promedio per cápita de hierro de $1.6 \mathrm{mg}$ en las familias de los quintiles más bajos, mientras que en las familias de los quintiles más ricos se observa una ingesta diaria per cápita de $3.6 \mathrm{mg}^{(5)}$. Un estudio realizado en Ayacucho, demostró que los niños de 6 a 23 meses tienen una ingesta reducida de hierro, zinc, calcio y niacina. Así, el $90 \%$ de los niños no alcanza los niveles recomendados de este nutriente ${ }^{(6)}$. Se describen hallazgos similares en Villa el Salvador al sur de Lima en niños de 6 a 11 meses $^{(7)}$. En Arequipa, provincia donde se realizó este estudio, la prevalencia de anemia en niños menores de tres años fue del $44,5 \%$ en $2016^{(4)}$.

Los sistemas de salud están muy interesados en optimizar las reservas de hierro del cuerpo humano en etapas muy tempranas del desarrollo infantil cuando hay un rápido desarrollo cerebral ${ }^{(8)}$. El desarrollo infantil es un proceso de cambios continuos. Las áreas del desarrollo infantil en las que se ha reconocido una fuerte asociación con la anemia incluyen el desarrollo mental, motor y conductual del individuo ${ }^{(9)}$. Por tanto, se han implementado intervenciones de suplementación con hierro en los sistemas sanitarios de nuestro país ${ }^{(1)}$. Sin embargo, la suplementación con hierro y micronutrientes requiere de estudios bien diseñados en Perú, ya que se ha demostrado una baja adherencia y, en consecuencia, una baja efectividad ${ }^{(10)}$.

Investigaciones anteriores coinciden en que el enriquecimiento de alimentos es la mejor estrategia a largo plazo en la prevención de la deficiencia de hierro en la población general ${ }^{(11)}$. Se sabe que la leche es una de las principales fuente de alimento en la infancia; la leche de vaca tiene una menor densidad y biodisponibilidad del hierro $^{(12,13)}$. Se recomienda lactancia materna exclusiva en los primeros seis meses de vida y su continuación hasta los veinticuatro meses de edad ${ }^{(3)}$. Las mezclas lácteos podrían ser una opción viable y sostenible para combatir la anemia en niños mayores de dos años. Sin embargo, los ensayos clínicos aleatorizados sobre el efecto de las mezclas lácteas fortificados con hierro y zinc sobre los niveles de hemoglobina son mínimos a nivel local. El estudio pretende cerrar la brecha de información existente para desarrollar una estrategia de control de la anemia en Perú.

En este contexto, nuestro estudio tiene como objetivo evaluar el efecto de la suplementación de una mezcla láctea fortificada con hierro-zinc sobre los niveles de hemoglobina e índices antropométricos en una población infantil de 2 a 10 años con o sin anemia leve.

\section{MATERIAL Y MÉTODO}

\section{Diseño}

Ensayo clínico aleatorizado, doble ciego y de grupos paralelos, controlado con mezcla láctea no fortificada con hierro y zinc.

\section{Participantes}

El estudio se realizó en el "Centro de Salud Juan Pablo II" en el distrito de Yura, provincia de Arequipa, un área rural y urbana ubicada a 2,590 metros sobre el nivel del mar (msnm) que cuenta con una población de 18,127 habitantes. En el área rural de Arequipa, solo el $53 \%$ de los jefes de hogar tienen educación primaria, el $29 \%$ son pobres y el $2 \%$ vive en pobreza extrema ${ }^{(14)}$. En la población infantil de menores de cinco años del distrito de Yura, el 68\% no cuenta con suplementos de micronutrientes de acuerdo con su edad. Según el Informe "Perú: consumo per cápita de los principales alimentos 2008 2009, INEI (2009), en el departamento de Arequipa se evidenció un consumo per cápita de $57,5 \mathrm{~kg} /$ persona de papa, 32,7 de pan, 30.2 de arroz, 18,9 de pollo, res 11,6 , pescado 3,9 y lácteos 12,5 . Siendo el distrito de Yura parte del departamento de Arequipa, podemos corroborar que coincide con los hábitos de consumo reportados por la población estudiada. Cabe mencionar que a la población estudiada se les brindó talleres y charlas de promoción de alimentación saludable. La población en estudio, no cuentan con agua potable ni desagüe.

Se incluyeron en el estudio niños de ambos sexos entre 2 y 10 años. Podían ingresar niños de 2-5 años con corrección de niveles de hemoglobina a $2.590 \mathrm{msnm} \geq 10 \mathrm{gr} / \mathrm{dL}$ y niños de 6 10 años con corrección de niveles de hemoglobina $\geq 11 \mathrm{gr} / \mathrm{dL}$ que también pudieran estar siguiendo tratamiento con hierro. El ajuste de los niveles de hemoglobina según la altura sobre el nivel del mar se realizó mediante la aplicación del factor de ajuste, cuya fórmula es: Nivel de hemoglobina ajustado = hemoglobina observada - factor de ajuste por altitud. Se excluyeron niños con desnutrición crónica, 
Revista del Cuerpo Médico del HNAAA, Vol 14 (3) - 2021

parasitosis intestinal, enfermedades crónicas, enfermedades congénitas, infecciones ocurridas la semana anterior al estudio, trastornos que requirieran una dieta especial (intolerancia o intoxicación alimentaria), dispepsia o estreñimiento, consumo de fármacos que pudieran interferir con la absorción de hierro, alergia o intolerancia conocida a los componentes de los productos lácteos, los que habían recibido otro suplemento nutricional y las familias que planeaban viajar en los primeros siete meses después del tratamiento.

\section{Tamaño de la muestra}

El tamaño de la muestra se basó en probar la hipótesis de que los niveles de hemoglobina eran más altos en el grupo que recibió la mezcla láctea fortificada con hierro y zinc que en el grupo que recibió la mezcla láctea no fortificada.

Estimamos que la magnitud de la diferencia para la relevancia clínica identificada fue de $1 \mathrm{gr} / \mathrm{dL}$ de hemoglobina para el grupo que recibió la mezcla láctea fortificada con hierro-zinc, con una desviación estándar de $1,2 \mathrm{gr} / \mathrm{dL}$, riesgo de error del $5 \%$, el poder estadístico del $90 \%$, hipótesis unilateral. Con estos parámetros el tamaño de muestra calculado fue de 64 niños. Sin embargo, se tuvo en cuenta un potencial de pérdida del seguimiento del $10 \%$, y finalmente se estimaron 72 participantes para el estudio. La población accesible estuvo constituida por 209 niños de 2 a 10 años, de los cuales 96 cumplieron los criterios de selección. En esta población elegible, 22 padres no aceptaron firmar un consentimiento informado, por lo que finalmente 67 niños fueron incluidos en el estudio.

\section{Aleatorización, ocultación de la asignación y cegamiento} Un total de 67 niños fueron inscritos y asignados al azar a uno de los dos grupos. El grupo experimental $(n=32)$ recibió la mezcla láctea fortificada con hierro y zinc, mientras que el grupo de control $(n=35)$ recibió la mezcla láctea no fortificada. El método utilizado para generar la secuencia de asignación aleatoria consistió en tablas de números aleatorios desarrolladas por un estadístico independiente que no participó en el estudio. Dado que no se podía acceder a la secuencia de asignación aleatoria; ni los participantes ni los investigadores del estudio conocían de antemano la intervención en la que se asignarían los sujetos. Las mezclas lácteas fueron administradas al grupo experimental y de control por nutricionista quien no conocía sobre la asignación de los participantes a los grupos de estudio. También se tuvieron en cuenta las características organolépticas de los productos lácteos, que eran muy similares. Las mezclas lácteas eran de color crema y tenían un olor y sabor característico, así como un aspecto uniforme. La presentación fue un recipiente metálico de 400 gramos para ambos grupos. Los investigadores estaban cegados a los resultados de los niveles de hemoglobina durante todo el estudio. Los familiares de los niños no sabían el tipo de mezcla láctea que estaban tomando sus hijos.

\section{Intervenciones y medidas}

Las mezclas lácteas fortificadas con hierro-zinc se administró al grupo experimental y la mezcla láctea no fortificada se administró al grupo de control.

Las dos mezclas lácteas se consideraron alternativas razonables al tratamiento nutricional de los niños, pero queríamos saber si la mezcla láctea enriquecida era superior para mejorar los niveles de hemoglobina.

Además del lácteo, los niños de ambos grupos que presentaban anemia leve al inicio de la intervención recibieron tratamiento con hierro (fumarato ferroso $3 \mathrm{mg} / \mathrm{Kg}$ ), que fue brindado por el personal del Centro de Salud Juan Pablo II en el distrito de Yura, Arequipa.

Las mezclas lácteas utilizadas en el estudio se obtuvieron por el financiamiento de una empresa productora y comercializadora de productos lácteos con registro sanitario otorgado por la Dirección General de Salud Ambiental del Perú.

El estudio de intervención dietética, que tuvo un seguimiento de 24 semanas, comenzó en junio de 2019 y finalizó en diciembre de 2019.

Al grupo experimental $(n=32)$ se le administraron $300 \mathrm{ml}(150$ gramos de la mezcla láctea enriquecida $+150 \mathrm{ml}$ de agua) dos veces al día. Esta mezcla láctea se obtuvo de la combinación de leche entera y otros ingredientes como maltodextrina de maíz, aceite de soja, suero de leche y minerales (pirofosfato de hierro y sulfato de zinc) enriquecidos con Vitaminas A y D.

Al grupo de control $(n=35)$ se le administraron $300 \mathrm{ml}(150$ gramos de la mezcla láctea no fortificada $+150 \mathrm{ml}$ de agua) dos veces al día. Esta mezcla láctea se obtuvo de la combinación de leche desnatada y suero de mantequilla con otros ingredientes como extracto de soja, maltodextrina y aceite vegetal enriquecido con vitaminas $A$ y $D$ y aditivos alimentarios autorizados. Ambas mezclas lácteas fueron pasteurizados, homogeneizados y enlatados en recipientes metálicos sanitarios para alimentos, sellados herméticamente y sometidos a un tratamiento de esterilización térmica para estar disponibles comercialmente. Los perfiles nutricionales detallados de ambos productos se muestran en la Tabla 1.

Los promotores de salud recibieron capacitación y participaron en talleres demostrativos sobre cómo preparar mezclas lácteas. La capacitación incluyó buenas prácticas de manipulación de alimentos, distribución de dosis, así como instrucciones para el cronograma de administración de las mezclas. La mezclas lácteas se dividieron en dos tomas diarias: por la mañana (entre las 9:30 y las 10:30 horas) y por la tarde (entre las 14:30 y las 15:30 horas). La secuencia de administración de las mezclas lácteas fue la misma tanto por la mañana como por la tarde; cada dosis consistió en (150 gramos de mezcla láctea con $150 \mathrm{ml}$ de agua); la mezcla láctea enriquecida aportó (381 Kcal 9,9 mg de hierro y 6,3 mg de zinc) y la mezcla láctea sin fortificar aportó (402 Kcal, sin aporte de hierro ni zinc) por día y se administró cinco días a la semana, durante 24 semanas.

Este procedimiento se registró en tarjetas y luego en un libro de registro dos veces al día. Los nombres completos de los niños, los códigos de identidad, la edad, el sexo, la residencia, los nombres de los familiares, el nombre de la institución educativa, los horarios de las fechas de administración, los efectos secundarios leves, los efectos 
adversos significativos, los niveles de hemoglobina y las mediciones antropométricas se registraron en las tarjetas al principio y fin del estudio. Para las mediciones antropométricas se utilizó una balanza Seca modelo 803 y una vara de medir estandarizada siguiendo los lineamientos del Instituto Nacional de Salud en Perú. Un antropometrista y un asistente realizaron estas mediciones.

Se realizó una reunión mensual con los padres de los niños para conocer la tolerancia y los probables efectos secundarios. Asimismo, se programaron contactos telefónicos y visitas semanales a los niños y sus familiares. Dos nutricionistas independientes realizaron el suministro de las mezclas lácteas en instituciones educativas, y la cantidad requerida se distribuyó mensualmente. Los dos grupos fueron monitoreados de manera similar. La supervisión de la administración de las mezclas lácteas fue realizada inesperadamente por los investigadores en cada punto de distribución tres veces por semana. Se revisaron los libros de registro de los niños de los grupos experimental y de control para evaluar si los dos grupos tenían una buena aceptación de las mezclas lácteas o no.

\section{Desenlaces}

\section{Desenlace primario}

Se eligió el nivel de hemoglobina en sangre como variable de respuesta o resultado principal. Se completaron tres mediciones de hemoglobina durante el seguimiento. La primera medición tuvo como objetivo determinar la prevalencia de anemia para seleccionar niños con o sin anemia leve para el estudio. Otras mediciones correspondientes al inicio y final del seguimiento se realizaron en julio, septiembre y diciembre. Los niveles de hemoglobina se obtuvieron mediante un muestreo capilar del lado lateral del dedo medio utilizando una lanceta estéril desechable (2 $\mathrm{mm}$ de longitud). Las muestras fueron analizadas en el laboratorio del centro de salud de Ciudad de Dios, Arequipa, utilizando una mini centrífuga BOECO HP240.

\section{Desenlaces secundarios}

Se evaluó el peso, la talla y el índice de masa corporal de los niños de 2 a 10 años al principio y al final del estudio.

\section{Análisis estadístico}

Todos los análisis se realizaron mediante el análisis por intención a tratar. Se tuvo en cuenta el efecto de la altitud para la corrección de los niveles de hemoglobina; el presente estudio se realizó a $2.590 \mathrm{msnm}$; el factor de ajuste para la altitud fue 1,3 utilizando la siguiente fórmula:

Nivel de hemoglobina ajustado = nivel de hemoglobina observado - factor de ajuste por altitud ${ }^{(15)}$.

Las variables numéricas se presentan como media y la desviación estándar (DE) y las variables categóricas se presentan como frecuencias y porcentajes. Las comparaciones entre las medias de hemoglobina se llevaron a cabo utilizando la prueba $\mathrm{t}$ de Student pareada para comparar las diferencias entre la línea de base y el punto final en cada grupo. Se utilizó la prueba de la t de Student para muestras independientes, cuyo objetivo fue analizar las diferencias entre los grupos de estudio al final del seguimiento. Las comparaciones entre variables categóricas se hicieron con la prueba Chi cuadrado. La significancia estadística tuvo un valor $\mathrm{p}<0,05$. Todos los análisis de datos se realizaron utilizando el software STATA, versión 14.0.

\section{Consideraciones éticas}

A los padres de los niños en edad preescolar y escolar que cumplieron con los criterios de selección se les solicitó firmar el consentimiento informado en diferentes aulas de las siete instituciones educativas involucradas en la investigación. El estudio se realizó de acuerdo con los lineamientos de la declaración de Helsinki, y todos los procedimientos que involucraron seres humanos fueron aprobados por el Comité de Ética del Hospital Nacional Alberto Sabogal, Lima, Perú (Carta N 185-CIEI-OFI \& DGRPS-HNASS-ESALUD -2019). Debido a que este ensayo clínico evalúa un alimento procesado, no se requirió su inscripción en el Registro de Ensayos Clínicos del Perú del Instituto Nacional de Salud.

\section{RESULTADOS}

Un total de 67 niños fueron asignados aleatoriamente a los dos grupos de estudio. De ellos, 32 niños fueron incluidos en el grupo experimental, mientras que 35 fueron incluidos en el grupo de control. En el seguimiento, tres y cinco niños no continuaron participando en el estudio debido a efectos secundarios leves como dolor abdominal intermitente y no pudieron asistir regularmente a la institución educativa. Tres niños no recibieron la intervención asignada en el grupo control por motivos personales (Figura 1). La población incluida en la línea de base del estudio tenía características similares en cuanto a edad, sexo, tamaño e índice de masa corporal. La edad promedio de los niños inscritos en el grupo experimental fue de 6,1 años $(D E=2,3)$ y la edad media en el grupo de control fue de 5,5 años $(D E=2,4)$. En cuanto al género, se incluyeron 16 hombres y 16 mujeres en el grupo

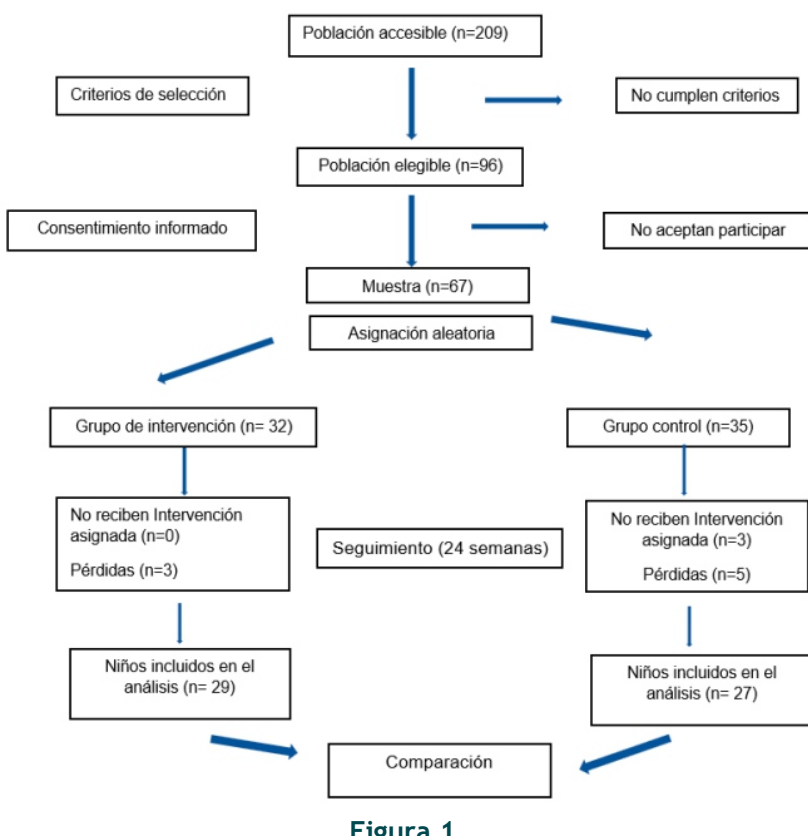

Asignación aleatoria y seguimiento del grupo experimental y grupo control 
Tabla 1. Composición nutritiva de las mezclas lácteas fortificada y no fortificada.

\begin{tabular}{|c|c|c|c|c|}
\hline Nutrientes & $\begin{array}{l}\text { Mezcla láctea } \\
\text { fortificada } \\
\text { (porción de } 100 \mathrm{~g} \text { ) }\end{array}$ & $\% \mathrm{RD}$ & $\begin{array}{c}\text { Mezcla láctea } \\
\text { no fortificada } \\
\text { (porción de } \\
100 \mathrm{~g} \text { ) }\end{array}$ & \%RD \\
\hline Energía (kcal) & 127 & & 134 & \\
\hline Grasa total (g) & 7 & 9 & 7,5 & 12 \\
\hline Grasa láctea (g) & 2,7 & & 2,0 & \\
\hline Grasa vegetal (g) & 4,3 & & 5,3 & \\
\hline Grasa saturada (mg) & 3,8 & 19 & 3,7 & 19 \\
\hline Grasas Trans (mg) & 0,1 & & 0,1 & \\
\hline Colesterol (mg) & 8 & 3 & 10 & 3 \\
\hline Sodio (mg) & 107 & 5 & 80 & 3 \\
\hline Carbohidratos totales (g) & 11,7 & 4 & 12,4 & 4 \\
\hline Fibra dietaria (g) & 0 & 0 & 0 & \\
\hline Azúcares totales (g) & 7 & 8 & 5,7 & \\
\hline Azúcares añadidos (g) & 0 & & 0 & \\
\hline Proteínas (g) & 4,2 & 8 & 3,9 & 8 \\
\hline Calcio (mg) & 153 & 15 & 170 & 17 \\
\hline Fósforo (mg) & 139 & 20 & 103 & 15 \\
\hline Hierro (mg) & 3,3 & 15 & 0 & 0 \\
\hline Zinc (mg) & 2,1 & 15 & 0 & \\
\hline Vitamina A (ugRE) & 240 & 30 & 240 & 30 \\
\hline Vitamina D (ug) & 1,5 & 30 & 1,5 & 30 \\
\hline
\end{tabular}

Fuente. Hoja Técnica. "Mezcla láctea compuesta con maltodextrina y aceite vegetal, enriquecida con vitaminas y minerales-Pura Vida". Hoja Técnica "Mezcla láctea compuesta con extracto de soya, maltodextrina, y aceite de soya-La Pradera" de la empresa fabricante.

Tabla 2. Características basales demográficas y clínicas para cada grupo.

\begin{tabular}{lcll} 
Variables & $\begin{array}{l}\text { Total } \\
(\mathrm{n}=67)\end{array}$ & $\begin{array}{l}\text { Experimental } \\
(\mathrm{n}=32)\end{array}$ & $\begin{array}{l}\text { Control } \\
(\mathrm{n}=35)\end{array}$ \\
\hline Edad (años) & $5,8(2,4)$ & $6,1(2,3)$ & $5,5(2,4)$ \\
Género (Masculino/Femenino) & $37 / 30$ & $16 / 16$ & $21 / 14$ \\
Peso $(\mathrm{Kg})$ & $21,1(7,2)$ & $23,0(8,2)$ & $19,3(5,8)$ \\
Talla $(\mathrm{m})$ & $1,1(0,1)$ & $1,1(0,1)$ & $1,1(0,1)$ \\
IMC $(\mathrm{Kg} / \mathrm{m} 2)$ & $17,0(2,0)$ & $17.5(2,2)$ & $16,5(1,8)$ \\
Hemoglobina (g/dL) & $11,7(0,6)$ & $11.8(0,5)$ & $11,5(0,7)$ \\
\hline Promedio (desviación estándar) & & &
\end{tabular}

Promedio (desviación estándar)

IMC, indice de masa corporal experimental, mientras que 21 y 14 se incluyeron en el grupo control. Al inicio del estudio, la concentración media de corrección de los niveles de hemoglobina a 2,590 msnm fue de $11,8 \mathrm{gr} / \mathrm{dL}(\mathrm{DE}=0,5)$ en el grupo experimental y de 11,5 $\mathrm{gr} / \mathrm{dL}(\mathrm{DE}=0,7),(\mathrm{p}=0,42)$ en el grupo de control (Tabla 2$)$.

Se evidenció un aumento de la concentración de hemoglobina en ambos grupos al final de la intervención, estadísticamente significativo. La hemoglobina aumentó en 1,31 $\mathrm{gr} / \mathrm{dL}$, $(p<0,001)$ en el grupo experimental, mientras que aumentó en $0,57 \mathrm{gr} / \mathrm{dL},(\mathrm{p}<0,001)$ en el grupo de control. Al final de la intervención, la hemoglobina fue de $13,1 \mathrm{gr} / \mathrm{dL}(\mathrm{DE}=0,9)$ en el grupo experimental y de $12,1 \mathrm{gr} / \mathrm{dL}(\mathrm{DE}=0,8)$ en el grupo control. Al final del estudio, la diferencias de medias entre ambos grupos fueron a favor del grupo experimental en 1.03 $\mathrm{gr} / \mathrm{dL}, \mathrm{DE}=0.21(\mathrm{p}<0.001)$ (Tabla 3).

En cuanto a los índices antropométricos al inicio del estudio, el peso promedio fue de $23 \mathrm{Kg}(\mathrm{DE}=8,2)$, talla 1,1 metros $(D E=0,1)$ e índice de masa corporal (IMC) de $17,5 \mathrm{Kg} / \mathrm{m} 2$ $(D E=2,2)$ en el grupo experimental, mientras que el peso medio fue de $19,3 \mathrm{Kg}(\mathrm{DE}=5,8)$, talla 1,1 metros $(D E=0,1)$ e IMC $16,5 \mathrm{Kg} / \mathrm{m} 2(\mathrm{DE}=1,8)$ en el grupo control (Tabla 2$)$.

Al final de la intervención, el peso aumentó en $3,09 \mathrm{Kg}$, $D E=2,37, \quad(p<0,001)$, la talla aumentó en 0,05 metros, $D E=0,02(p<0,001)$ y el IMC aumentó en $0,76 \mathrm{~kg} / \mathrm{m} 2(\mathrm{DE}=1,42)$ $(p<0,005)$ en el grupo experimental. En el grupo de control, también hubo cambios en las medidas antropométricas. El peso aumentó en $1,95 \mathrm{Kg}(\mathrm{DE}=2,04)(\mathrm{p}<0,001)$, el tamaño fue el mismo y el IMC aumentó en $0,38 \mathrm{Kg} / \mathrm{m} 2 \quad(D E=0,93)$ $(p<0,001)$. Al final del estudio, se observaron diferencias en ambos grupos con respecto a las siguientes variables: peso: $4,84 \mathrm{Kg}(\mathrm{DE}=2,00)(\mathrm{p}=0,001)$, talla: 0,07 metros, $\mathrm{DE}=0,03$ $(p=0,045)$ e IMC: $1,28 \mathrm{Kg} / \mathrm{m2}(D E=0,59)(p=0,032)$. Estas diferencias fueron estadísticamente significativas en el grupo experimental (Tabla 3).

\section{DISCUSIÓN}

Este estudio es el primer ensayo clínico aleatorizado, doble ciego y controlado, realizado con niños nutricionalmente vulnerables de 2 a 10 años en un área distante con escasos recursos económicos ubicada a 2.590 metros sobre el nivel del mar en el distrito de Yura, Arequipa, Perú. Nuestros

Tabla 3. Diferencias basal y al final del seguimiento para las variables de resultado entre los grupos de estudio.

\begin{tabular}{|c|c|c|c|c|c|c|c|c|}
\hline Variables & $\begin{array}{l}\text { Diferencia } \\
\text { total }\end{array}$ & Valor $p^{a}$ & $\begin{array}{l}\text { Diferencia entre } \\
\text { el grupo } \\
\text { experimental }\end{array}$ & Valor $\mathrm{p}^{\mathrm{a}}$ & $\begin{array}{l}\text { Diferencia entre } \\
\text { el grupo control }\end{array}$ & Valor $\mathrm{p}^{\mathrm{a}}$ & $\begin{array}{l}\text { Diferencia entre los } \\
\text { grupos al termino de } \\
\text { seguimiento }\end{array}$ & Valor $p^{b}$ \\
\hline Hemoglobina (g/dL) & $0,92(0,93)$ & $<0,001$ & $1,31(0,92)$ & $<0,001$ & $0,57(0,81)$ & $<0,001$ & $1,03(0,21)$ & $<0,001$ \\
\hline Peso $(\mathrm{Kg})$ & $2,49(2,26)$ & $<0,001$ & $3,09(2,37)$ & $<0,001$ & $1,95(2,04)$ & $<0,001$ & $4,84(2,00)$ & $<0,001$ \\
\hline Talla (m) & $0,04(0,02)$ & $<0,001$ & $0,05(0,02)$ & $<0,001$ & $0,04(0,03)$ & $<0,001$ & $0,07(0,03)$ & 0,045 \\
\hline
\end{tabular}

Promedio (Desviación estándar)

IMC, indice de masa corporal

at de Student para muestras pareadas

$b t$ de Student para muestras independientes 
Revista del Cuerpo Médico del HNAAA, Vol 14 (3) - 2021

resultados muestran que la ingesta diaria del mezcla láctea fortificada con $9,9 \mathrm{mg}$ de pirofosfato de hierro y $6,3 \mathrm{mg}$ sulfato de zinc dividido en dos tomas diarias durante 24 semanas mejoró los niveles de hemoglobina y los índices antropométricos.

La magnitud del impacto sobre la hemoglobina identificada en nuestro estudio fue consistente con una revisión sistemática de ensayos controlados aleatorizados que demuestran que la suplementación con hierro fortificado las fórmulas a base de leche aumentan significativamente los niveles de hemoglobina en los niños, con un aumento en la concentración de hemoglobina ${ }^{(16)}$.

Una revisión sistemática evaluó el efecto de mezclas lácteas fortificadas mostrándose que tuvo efectos mínimos sobre el aumento de peso; el riesgo de anemia se redujo en los grupos de leche fortificada y no hubo efectos significativos sobre el aumento de altura, los cambios en la composición corporal o la concentración de hemoglobina ${ }^{(17)}$. Otra revisión sistemática de ensayos clínicos demostró que en Asia, África y el Caribe la fortificación con micronutrientes en el hogar en comparación con ninguna intervención o placebo redujo el riesgo de anemia y la deficiencia de hierro en bebés y niños pequeños ${ }^{(18)}$. En contraste una revisión sistemática incluyó 24 ensayos controlados aleatorizados donde se encontró un aumento muy pequeño pero no significativo de los valores de hemoglobina con los productos lácteos enriquecidos con micronutrientes en la salud de los niños y adolescentes (5-15 años) ${ }^{(19)}$.

Se observó una diferencia positiva entre los grupos experimental y control al final de la intervención con respecto a los índices antropométricos. Una revisión sistemática que utilizó un producto lácteo fortificado con hierro, zinc y otros nutrientes informó efectos mínimos sobre el peso; sin embargo, se observó un pequeño aumento en comparación con el grupo de control y no se observaron efectos significativos sobre el aumento de estatura ${ }^{(17)}$. Nuestros resultados positivos probablemente estén influenciados por la duración de la intervención y el bajo nivel socioeconómico de la población de estudio. Tras la intervención, el peso aumentó en ambos grupos de estudio, mostrando mayores proporciones en el grupo experimental. Una posible causa del aumento más significativo en el grupo experimental podría ser que, en este grupo, la mezcla láctea fortificada con zinc ha mejorado la función biológica del sentido del gusto y el apetito ${ }^{(20)}$.

La absorción de hierro de los alimentos enriquecidos con hierro debe estar regulada por el estado nutricional del hierro en el niño. En este estudio, incluimos niños con riesgo de presentar anemia y niños con anemia leve para evitar el riesgo potencial de sobrecarga de hierro. La mezcla láctea fortificada se reconstituyó con agua sin alterar la biodisponibilidad de los micronutrientes ${ }^{(21)}$. Las mezclas lácteas administradas se fortificaron con 3,3 mg Fe y 2,1 mg Zn por cada gramo de mezcla láctea. En este sentido, un estudio dirigido a determinar el efecto del zinc sobre la absorción de hierro de la leche fortificada concluye que la adición de zinc a $20 \mathrm{mg} / \mathrm{L}$ no inhibe significativamente la absorción de hierro de la leche fortificada con $10 \mathrm{mg} / \mathrm{L}$ de hierro ${ }^{(22)}$. Asimismo, otro estudio afirma que la relación entre hierro y zinc no debe ser mayor a dos para evitar una reducción de la biodisponibilidad del zinc. En nuestro estudio, la relación entre el hierro y el zinc fue de $1,57^{(23)}$.

El enriquecimiento de alimentos es uno de los métodos más efectivos para prevenir la deficiencia de hierro en los niños; por lo tanto, para su implementación se debe seleccionar un alimento específico (vehículo) que el grupo destinatario pueda consumir con mucha frecuencia. Las mezclas lácteas son un excelente vehículo para la suplementación de hierro en los niños. En este ensayo clínico, se administró a niños una mezcla láctea fortificada con hierro y zinc con buena aceptación sensorial, que estaba disponible comercialmente en Perú. Un estudio realizado en Turquía informa que la suplementación láctea con micronutrientes puede ser preferible debido a su fácil uso y bajo costo ${ }^{(23)}$.

La deficiencia de hierro es la principal causa de anemia en todas las edades, principalmente prevalente en niños escolares de familias pobres de Indonesia ${ }^{(24)}$. En nuestro país también es más prevalente especialmente en las familias pobres de las zonas rurales ${ }^{(5)}$

En cuanto a la adherencia, el vehículo utilizado para la suplementación mostró niveles adecuados de adherencia en ambos grupos de estudio. En este sentido, experiencias previas con otros vehículos utilizados para la suplementación no demostraron efecto sobre los niveles de hemoglobina. En un estudio realizado en Apurímac, Perú, la suplementación con micronutrientes administrada en sobres denominados Chispitas no encontró asociación entre el número de sobres recibidos o tomados y la anemia. Los autores concluyeron que la dispensación o ingesta de la cantidad necesaria de micronutrientes no es suficiente, pero se debe garantizar un proceso de ingesta equitativo para reducir la prevalencia de anemia. Este punto de vista debe tenerse en cuenta para mejorar esta intervención ${ }^{(25)}$. Los gestores sanitarios de nuestro país deben considerar que la leche fortificada podría ser una nueva alternativa para planificar políticas públicas relacionadas con la suplementación con hierro y zinc en niños por sus altos valores nutricionales y buena calidad sensorial.

Nuestro estudio tiene limitaciones, durante el seguimiento, hubo tres abandonos en el grupo experimental y ocho en el grupo control porque los niños no pudieron asistir a la escuela por problemas personales. A pesar del pequeño tamaño de la población de estudio, nuestros hallazgos son consistentes con los hallazgos de estudios anteriores. Debido al uso de una población en situación de pobreza, nuestros resultados no son generalizables a otras partes del país. Sin embargo, más estudios deberían intentar romper estas limitaciones.

En conclusión, nuestro estudio demuestra que la administración de mezclas lácteas fortificadas con hierrozinc es una opción viable que provoca un efecto positivo en los niveles de hemoglobina en niños en edad preescolar y escolar.

\section{REFERENCIAS BIBLIOGRÁFICAS}

1. Francke P, Acosta G. Impacto de la suplementación con micronutrientes sobre la desnutrición crónica infantil en Perú. Rev Medica Hered. [Internet]. 2020 Oct 16;31(3):148-54. Available from: http: //www.scielo.org.pe/scielo.php?script=sci_arttext\&pid=S1018 
$-130 \times 2020000300148 \& \operatorname{lng}=e s \& n r m=i s o \& t \operatorname{lng}=e s$

2. Cappellini MD, Musallam KM, Taher AT. Iron deficiency anaemia revisited. J Intern Med [Internet]. 2020 Feb 1;287(2):153-70. Available from: https://pubmed.ncbi.nlm.nih.gov/31665543/

3. Ministerio de Salud. Reglamento de Alimentación Infantil [Internet]. L i m a ; $2019 \mathrm{Nov}$. A v a i l a b l e f r o m: http://bvs.minsa.gob.pe/local/MINSA/4922.pdf

4. Instituto Nacional de Estadística e Informatica. Encuesta Demográfica y de Salud Familiar 2016 Nacional y Regional (ENDES 2016 ). Lima: INEI. 2017 . Available from https://www.inei.gob.pe/media/MenuRecursivo/publicaciones_dig itales/Est/Lib1433/index.htm

5. Ministerio de Salud. Plan Nacional para la Reducción y Control de la Anemia Materno Infantil y la desnutrición Crónica Infantil en el Perú: $2017-2021$. Lima; 2017 . Ava il able from: http://bvs.minsa.gob.pe/local/MINSA/4189.pdf

6. Fundación contra el Hambre. Aproximación al consumo de alimentos y prácticas de alimentación y cuidado infantil en niños y niñas de 6 a 23 meses de edad realizado en familias de la provincia de Vilcashuamán, región Ayacucho Fundación Acción Contra el Hambre. 2012; Available from: http://catalogocindoc.ins.gob.pe/cgibin/koha/opac-detail.pl?biblionumber $=10328$

7. Zavaleta N, Kvistgaard AS, Graverholt G, Respicio G, Guija H, Valencia N, et al. Efficacy of an MFGM-enriched Complementary Food in Diarrhea, Anemia, and Micronutrient Status in Infants. J Pediatr Gastroenterol Nutr [Internet]. 2011 Nov;53(5):561-8. Available from: https: / /journals.Iww.com/00005176-201111000-00020

8. Larson LM, Phiri KS, Pasricha S-R. Iron and Cognitive Development: What Is the Evidence? Ann Nutr Metab [Internet]. 2017 Dec 1;71 Suppl 3(3):2 $5-38$. A v a il a ble f $\mathrm{rom}$ : https://pubmed.ncbi.nlm.nih.gov/29268256/

9. Zavaleta N, Astete-Robilliard L. Effect of anemia on child development: Long-term consequences. Rev Peru Med Exp Salud Publica [Internet]. 2017 Oct 1;34(4):716-22. Available from: https: / / pubmed.ncbi.nlm.nih.gov/29364424/

10. Aparco Balboa JP, Huamán-Espino L. Recommendations for interventions with iron supplements: Lessons learned in a community trial in four regions of Peru. Rev Peru Med Exp Salud Publica [Internet]. 2017 Oct 1;34(4):709-15. Available from: https://pubmed.ncbi.nlm.nih.gov/29364410/

11. Nikooyeh B, Neyestani TR. Effectiveness of various methods of home fortification in under-5 children: where they work, where they do not. A systematic review and meta-analysis. Nutr Rev [Internet]. 2021 A p r 1;79 (4):445-61. Available from https://pubmed.ncbi.nlm.nih.gov/33011799/

12. Fransson G-B, Lönnerdal B. Distribution of Trace Elements and Minerals in Human and Cow's Milk. Pediatr Res [Internet]. $1983 ; 17(11): 912-5$. A v a il a ble f rom: https://pubmed.ncbi.nlm.nih.gov/6646903/

13. Oliveira MAA, Osório MM. Cow's milk consumption and iron deficiency anemia in children. Vol. 81, Jornal de Pediatria. J Pediatr (Rio J) [Internet]. 2005 Sep [cited 2021 Sep 13];81(5):361-7. Available from: https: / /pubmed.ncbi.nlm.nih.gov/16247536/

14. Instituto Nacional de Estadística e Informática. Arequipa Compendio Estadístico Regional 2011. Lima: INEI. Available from: https://www.inei.gob.pe/media/MenuRecursivo/publicaciones_dig itales/Est/Lib1433/index.html
15. Sullivan KM, Mei Z, Grummer-Strawn L, Parvanta I. Haemoglobin adjustments to define anaemia. Trop Med Int Heal [Internet]. 2008 Oct ; $13(10): 1267-71$. Ava il a ble from. https://pubmed.ncbi.nlm.nih.gov/18721184/

16. Gera T, Sachdev HPS, Nestel P, Sachdev SS. Effect of Iron Supplementation on Haemoglobin Response in Children: Systematic Review of Randomised Controlled Trials. J Pediatr Gastroenterol Nutr [Internet]. 2007 Apr;44(4):468-86. Available from: https://pubmed.ncbi.nlm.nih.gov/17414146/

17. Matsuyama M, Harb T, David M, Davies PSW, Hill RJ. Effect of fortified milk on growth and nutritional status in young children: a systematic review and meta-analysis. Public Health Nutr [Internet]. 2017 May $1 ; 20(7): 1214-25$. A v a i l a ble from : https: //pubmed.ncbi.nlm.nih.gov/27938461/

18. Suchdev PS, Jefferds MED, Ota E, da Silva Lopes K, De-Regil LM. Home fortification of foods with multiple micronutrient powders for health and nutrition in children under two years of age. Cochrane Database Syst Rev [Internet]. 2011 Sep 7;2011(9). Available from: https: //pubmed.ncbi.nlm.nih.gov/21901727/

19. Eichler K, Hess S, Twerenbold C, Sabatier M, Meier F, Wieser S. Health effects of micronutrient fortified dairy products and cereal food for children and adolescents: A systematic review. PLoS One [Internet]. 2019 Jan 1; 14 (1). A v a i l a ble from : https://pubmed.ncbi.nlm.nih.gov/30673769/

20. Chao HC, Chang YJ, Huang WL. Cut-off Serum Zinc Concentration Affecting the Appetite, Growth, and Nutrition Status of Undernourished Children Supplemented With Zinc. Nutr Clin Pract [Internet]. 2018 Oct 1;33(5):701-10. Available from: https://pubmed.ncbi.nlm.nih.gov/29603391/

21. Olivares M, Hertrampf E, Pizarro F. Effect of iron stores on heme iron absorption. Nutr Res [Internet]. 1993 Jun 1;13(6):633-8. Available

https://www.sciencedirect.com/science/article/abs/pii/S0271531 70580555X\#: :text=These suggest that heme iron,0.66\%2C p\%3D0.00001).

22. Olivares M, Wiedeman A, Bolívar L, López de Romaña D, Pizarro F. Effect of Increasing Concentrations of Zinc on the Absorption of Iron from Iron-Fortified Milk. Biol Trace Elem Res. 2012;150(1-3):21-5.

23. Biringen Löker G, Ugur M, Yıldız M. A parrtial supplementation of pasteurized milk with vitamin $\mathrm{C}$, iron and zinc. Nahrung/Food [Internet]. 2003 Feb;47(1):17-20. Available from: https://pubmed.ncbi.nlm.nih.gov/12653430/

24. Bardosono S, Dewi LE, Sukmaniah S, Permadhi I, Eka AD, Lestarina L. Effect of a six-month iron-zinc fortified milk supplementation on nutritional status, physical capacity and speed learning process in Indonesian underweight schoolchildren: randomized, placebocontrolled. Med J Indones [Internet]. 2009 Aug 1 [cited 2021 Sep 13 ] ; 18 ( 3 ) : $193-202$. A v a i l a ble f r o m : https://mji.ui.ac.id/journal/index.php/mji/article/view/361

25. Huamán-Espino L, Aparco JP, Nuñez-Robles E, Gonzáles E, Pillaca J, Mayta-Tristán P. Consumption of Chispitas ${ }^{\circledR}$ Multimicronutrient supplements and anemia in 6 - 35-month-old children: Cross-cut study in the context of a populational health intervention in Apurimac, Peru. Rev Peru Med Exp Salud Publica [Internet]. 2012 Aug $29 ; 29(3): 314-23$. A v a i l a ble from : http: / /www.scielo.org.pe/scielo.php?script=sci_arttext\&pid=S1726 $-46342012000300004$ 